\title{
TAKING GREEN COMPUTING TO THE COMPUTER LAB
}

\author{
Philip Robertson, Georgia College,philip.robertson1@bobcats.gcsu.edu \\ Bryan Marshall, Georgia College, bryan.marshall@gcsu.edu \\ Peter W. Cardon, University of Southern California, cardon@marshall.usc.edu \\ Natalya Goreva, Point Park University, ngoreva@pointpark.edu
}

\begin{abstract}
Desktop virtualization has become an increasingly popular option to organizations looking to "go green." In this research paper, we describe the process we took to go green in one of the computer labs on our campus. We identify the financial and other benefits of going green.
\end{abstract}

Keywords: Sustainability, Information Technology (IT), Green Computing, Desktop Virtualization

\section{INTRODUCTION}

In today's world, organizations are constantly searching for ways to conduct their operations in an environmentally responsible manner. Nearly all organizations use computing devices to achieve their goals, including desktop computers that use a great deal of energy. Thin clients, which are computing devices designed to reduce power consumption, are becoming a more viable option compared to desktop computers. Utilizing a virtual environment and employing thin-client workstations should reduce the energy consumption and environmental impact of an organization [3].

The purpose of this paper is to identify the risks and benefits to organizations seeking to utilize green computing. Although there are many aspects associated with green computing, this paper will focus on the hardware and infrastructures needed for an organization to achieve cost and power savings.

\section{LITERATURE REVIEW}

This review has three purposes: define green computing; describe related challenges and benefits; and provide possible methods for extracting its power. Green computing is the study and practice of designing, manufacturing, using, and disposing of computers, servers, and associated subsystems - such as monitors, printers, storage devices, and networking and communications systems - efficiently and effectively with minimal or no impact on the environment [7]. With regard to the focus of this paper, green computing will refer to utilizing energy-efficient computing devices in an attempt to reduce energy usage while ideally lowering energy costs.

To help cut costs, many firms have eyed green strategies as a means to improve their bottom line [8]. From large universities to small businesses, the gains realized by an organization utilizing green initiatives can be vast. By replacing large, power hungry desktop machines with tiny, optimized thin clients, the carbon footprint generated can be reduced dramatically. A thin client is a computer which relies on another computer (usually a server) to perform the processing operations that a normal computer does alone. If an organization can replace even a handful of desktop computers with thin clients, power consumption should be reduced, resulting in energy savings and lower emissions. An additional benefit is the reduced hardware expenses. One desktop computer costs roughly the same as four thin clients. In one example, an elementary school that couldn't afford to buy more than two computers per classroom, decided to utilize thin clients in place of traditional desktop computers. Each classroom now has four thin client workstations that are powered by a single Windows-powered machine [2].

As for maintenance, there are few reasons to service or upgrade any of the internal components of thin client terminals. It's all done in a virtual environment that can be serviced from literally anywhere in the world. Organizations can further reduce expenses by outsourcing part or all of their IT staff to off-site support services [5]. 


\section{Issues in Information Systems}

Volume 13, Issue 1, pp. 294-299, 2012

By utilizing server virtualization and thin clients, larger businesses can reduce their energy consumption by up to $80 \%$ and increase hardware utilization levels to nearly $85 \%$ [6]. The most common type of a thin client is a computer terminal, which is responsible for channeling input and output signals to the server. To help illustrate this idea, think of your cable or satellite box at home. It doesn't generate the shows or movies that you watch but instead relies on the information being processed by your provider. It basically acts as the intermediary. It only provides your TV with the information you requested. The server accepts the information requests from the user, processes those requests, and then sends the information back to the thin client to be displayed to the user. The end user experiences this in the same way they do when using traditional computers. Ideally, the user would be unaware that the processing portion is being handled externally.

In one study, technology supervisors from five different schools were interviewed individually. Each interviewee explained their reasons for making the switch, the benefits realized, and the problems they faced. Virtualization allowed these schools to manage the massive increase in computing devices while surviving both budget and staffing cuts [2]. With regard to security, it is an extremely important factor in pushing the agenda for desktop virtualization. Secondly, IT staffs are being asked to deliver more with fewer resources. While companies are looking to add more desktops for a growing user population, IT departments are asked to support this demand with what they already have. Desktop virtualization provides an answer to those in need [4].

Green computing offers many benefits, ranging from reduced energy costs to increased productivity of the IT department. One benefit in particular is a reduction in time spent installing new machines and software. In one example, a school that instituted virtualization saved many hours, if not days, by installing an application once and pushing it out to each system [2]. IBM has been able to reduce power and cooling, maintenance, software support, and personal costs by nearly $70 \%$ through implementing virtualization [1].

By utilizing virtualization, multiple virtual computers with their own configurations can run simultaneously on a single physical server. This is achieved while guaranteeing each virtual computer receives the resources it needs [5].Through this consolidation, hardware resources are maximized, and the need for multiple physical servers is mitigated. Furthermore, with reductions in servers, there is a corresponding reduction in space, power, cooling, and maintenance, reducing plant running costs [6]. Over $70 \%$ of virtualization projects successfully realize important benefits.

\section{Challenges}

As with any new technology, there are a number of challenges that users experience at first. With the right support system in place though, these problems can be addressed and resolved given the proper resources, knowledge, and timeframe. A Pennsylvania school's technology supervisor initially experienced problems with memory allocation. Reyer stated that "if you don't have virtual servers sized right, they do not quit gracefully." Reyer learned early on that if you don't allocate memory properly or have enough overhead, servers quit suddenly and with no warning [2]. This can lead to the entire virtualization system failing until the servers are restarted. In addition, virtualization technology is fully dependent on network transmissions. If the network goes down for any reason, the terminals will not function. This could be addressed by making sure that the network hardware is up to date and running smoothly. When utilizing virtualization, network load will increase, so it's a good idea to make sure ahead of time that the current layout can support the new technology. [7]

Lastly, it can be difficult for IT managers to see an immediate return on investment for their facility by utilizing virtualization. They understand the need to ensure hardware reliability and the ability to receive support on the operating systems. However, this is viewed as an unnecessary disruption that may have an impact that doesn't equal the expected outcome [6]. 


\section{Issues in Information Systems}

Volume 13, Issue 1, pp. 294-299, 2012

\section{RESEARCH METHODOLOGY}

In this research paper we decided to focus on the following three research questions. First, what are the financial benefits of adopting green technology in the lab? Second, are there any other benefits found from using the technology? And finally, are there any disadvantages in using the technology?

The first thing we did was set up the new lab. The lab that we replaced had eight Dell Optiplex GX520 workstations. We replaced each of these workstations with N-Computing L300 thin clients connecting to a Dell PowerEdge R415 Server. When converting the lab from desktop computers to thin client devices, we were able to leave all existing hardware in place. This included monitors, network cables and hardware, keyboards, mice, and a printer. This allowed us to properly isolate and credit any changes in performance and power usage to their proper source.

The server was installed in an existing rack and loaded with the VMWare ESX 4.0 software. We then created a new virtual appliance using Windows 7 Professional. The N-Computing thin client management software, known as vSpace, was then configured on the Windows 7 appliance via a remote desktop session. Once completed, the thin clients were then connected to the server via the network. After the initial configuration was completed, it was then possible to log into each thin client using an account created on the Windows 7 appliance.

\section{Financial Savings from Power Consumption}

By monitoring the power usage of the computing devices, we were able to estimate energy savings for organizations adopting desktop virtualization. In order to measure each device's power consumption, we installed a Kill-A-Watt P4400 metering device between the unit's power supply and electrical outlet. This provided us with the amount of wattage the devices were drawing in real time. Measurements were taken while the devices were idle as well as under a 90 percent load.

Once the thin client computers were configured properly, we conducted the power monitoring tests that were conducted on the Dell desktop computers. Due to the fact that the thin clients depend on the server to operate, we also measured power usage of the server. We then averaged the power consumption to generate a more realistic and comparable figure.

In order to simulate a 90 percent load, PassMark's Performance Test software was installed and executed on each configuration. Our goal was to accurately capture energy usage in real time under different levels of CPU utilization. Once we collected enough data from each device, we then compared the results and analyzed our findings. By measuring the amount of energy each type of configuration uses, we were then able to estimate energy savings over a period of one year.

The recorded energy usage from each device is shown in Table 1. The kilowatt hours calculated reflect business days, weekends, and holidays. During business days, the computers were under load for about six hours, and remained idle the remaining eighteen hours of the day. During the weekend and on holidays, the computers remained idle for the full day.

Table 1. Energy Consumption

\begin{tabular}{|l|c|c|c|c|}
\hline Device & $\begin{array}{c}\text { Average wattage } \\
\text { while idle }\end{array}$ & $\begin{array}{c}\text { Average wattage } \\
\text { under load }\end{array}$ & $\begin{array}{c}\text { Kilowatt hours per } \\
\text { business day }\end{array}$ & $\begin{array}{c}\text { Kilowatt hours per } \\
\text { weekend day \& holiday }\end{array}$ \\
\hline Dell Desktop & 90 & 118 & 2.328 & 2.16 \\
\hline $\begin{array}{l}\text { N-Computing } \\
\text { Thin Client }\end{array}$ & 5.5 & 5.7 & .1332 & .132 \\
\hline $\begin{array}{l}\text { Dell PowerEdge } \\
\text { Server }\end{array}$ & 140 & 150 & 3.42 & 3.36 \\
\hline
\end{tabular}




\section{Issues in Information Systems}

Volume 13, Issue 1, pp. 294-299, 2012

Assuming that each year contains 251 working days and 114 weekend and holiday days, we calculated the estimated kilowatt hours needed per year to run the units. We used a rate of $\$ 0.15$ per kilowatt hour to calculate the yearly costs. Table 2 depicts these calculations.

We wanted to determine whether incorporating desktop virtualization would lead to a reduction in energy use. As shown below, when combining the efforts of a server and thin clients, desktop virtualization did lead to a reduction in energy use.

Table 2. Estimated Yearly Energy Use and Costs for Computer Lab with 8 and 32 Workstations

\begin{tabular}{|l|c|c|}
\hline & $\begin{array}{c}\text { Kilowatt } \\
\text { hours per } \\
\text { Year }\end{array}$ & $\begin{array}{c}\text { Yearly } \\
\text { Cost }\end{array}$ \\
\hline Device Costs & & \\
\hline Dell Desktop & 832.73 & $\$ 124.91$ \\
\hline Thin Client & 48.61 & $\$ 7.29$ \\
\hline Dell PowerEdge Server & 1244.82 & $\$ 186.72$ \\
\hline Non-Virtualization Scenario & & \\
\hline 8 Workstations: 8 Dell Desktops & 6661.84 & $\$ 999.27$ \\
\hline 32 Workstations: 32 Dell Desktops & 26647.36 & $\$ 3997.08$ \\
\hline Virtualization Scenario & & \\
\hline 8 Workstations: 8 Thin Clients \& 1 Dell PowerEdge Server Combination & 1633.7 & $\$ 245.06$ \\
\hline 32 Workstations: 32 Thin Clients \& 1 Dell PowerEdge Server Combination & 2800.34 & $\$ 420.05$ \\
\hline
\end{tabular}

Estimates were provided for eight workstations as well as 32. In the eight-workstation scenario, we estimate that we would save $\$ 754.21$ per year in energy costs and reduce energy use by 5,028 kilowatt hours per year. In the 32workstation scenario, we estimate we could save $\$ 5,028.14$ per year in energy costs and reduce energy use by 23,847 kilowatt hours per year. These are dramatic differences: a $75.5 \%$ drop in costs and an $89.5 \%$ lower use of energy.

\section{Other Advantages}

The biggest advantage we found dealt with the IT and administration side of the workstations. When using the management console, each workstation could receive new software and updates simultaneously, decreasing the time normally spent when upgrading them individually. We were also able to monitor what the activity of the students by viewing them in real time.

\section{Disadvantages}

The only drawback we found in this configuration was the lack of 3D support from the video drivers. This is mainly due to the intensity of rendering display graphics. In a traditional computer, a video card is physically connected to the motherboard and directly linked to the computers processor. In a thin client configuration, the video processing takes place on the server, which then has to be fed back to the client via network devices. This prevents some of the more with graphic-intensive applications like Adobe Photoshop from running as well as they should. For the majority of internet and word processing applications, students encountered no issues.

\section{CONCLUSIONS}

Incorporating desktop virtualization offers many advantages over standard computing systems. The benefits were not only limited to energy and cost savings; performance increased as well. While there can be difficulties when first initializing and setting up a virtual network, the benefits and rewards can far outweigh these problems when executed properly. Having a competent IT staff or specialized team to oversee the initialization of the environment can go a long way in extracting the maximum utilization of your virtual network. Based upon the results of our 


\section{Issues in Information Systems}

Volume 13, Issue 1, pp. 294-299, 2012

study, we concluded that significant energy and cost savings can be realized when incorporating green computing methods.

\section{REFERENCES}

1. Anderson, D. (2008). IBM "Green Computing". IBM Corporation.

2. Anonymous. (2010). More bang for your bucks. Tech \& Learning, 31(3), 40-42

3. Anonymous. (2009). Green IT: Why Mid-size Companies Are Investing Now. Info-Tech Research Group.

4. Express, I. (2010). Desktop virtualization: The virtual desktop. Express Computer.

5. Hodge, P. (2011). Virtualization 101: Understanding how to do more with less. INTECH, 58(4), 28-30.

6. Manjari, J. (2010). Save energy by eliminating server underutilization. Express Computer.

7. Murugesan, S. (2008). Harnessing Green IT: Principles and Practices. IT Professional, 24-33.

8. Ostrowski, S. (2011). Green IT trending upward as a priority for organizations. Targeted News Service. 


\section{Issues in Information Systems}

\section{APPENDIX}

Specifications for devices:

\section{Desktop Computer}

\begin{tabular}{|l|l|}
\hline Brand & Dell \\
\hline Model & OptiPlex GX 520 \\
\hline Memory & 2 GB \\
\hline Processor & Pentium D CPU 3.00 GHz \\
\hline Hard Drive Size & 40 GB \\
\hline Operating System & Windows XP Service Pack 3 \\
\hline
\end{tabular}

\section{Thin Client}

\begin{tabular}{|l|l|}
\hline Brand & N-Computing \\
\hline Model & L300 \\
\hline Memory & Shared 24 GB of RAM \\
\hline Processor & Shared Dual Processors \\
\hline Hard Drive Size & Shared 500 GB \\
\hline Operating System & Windows 7 Professional \\
\hline
\end{tabular}

\section{Server}

\begin{tabular}{|l|l|}
\hline Brand & Dell \\
\hline Model & PowerEdge R415 \\
\hline Memory & 32 GB \\
\hline Processor & AMD Opteron 4130 2.6GHZ Dual Processors \\
\hline Hard Drive Size & 500GB \\
\hline Operating System & VMWare ESX 4.0 \\
\hline
\end{tabular}

